\title{
Les médiateurs scientifiques en Europe : une diversité de pratiques, une communauté de besoins
}

Scientific mediators in Europe: a diversity of practices, a community of needs

\section{Olivier Richard et Sarah Barrett}

\section{QpenEdition}

\section{Journals}

Édition électronique

URL : http://journals.openedition.org/ocim/862

DOI : $10.4000 /$ ocim. 862

ISSN : 2108-646X

Éditeur

OCIM

Édition imprimée

Date de publication : 1 mai 2011

Pagination : 5-12

ISSN : 0994-1908

\section{Référence électronique}

Olivier Richard et Sarah Barrett, «Les médiateurs scientifiques en Europe : une diversité de pratiques, une communauté de besoins », La Lettre de l'OCIM [En ligne], 135| 2011, mis en ligne le 01 mai 2013, consulté le 19 avril 2019. URL : http://journals.openedition.org/ocim/862 ; DOI : 10.4000/ocim.862 


\title{
Les médiateurs scientifiques en Europe : une diversité de pratiques, une communauté de besoins
}

\author{
Olivier Richard et Sarah Barrett *
}

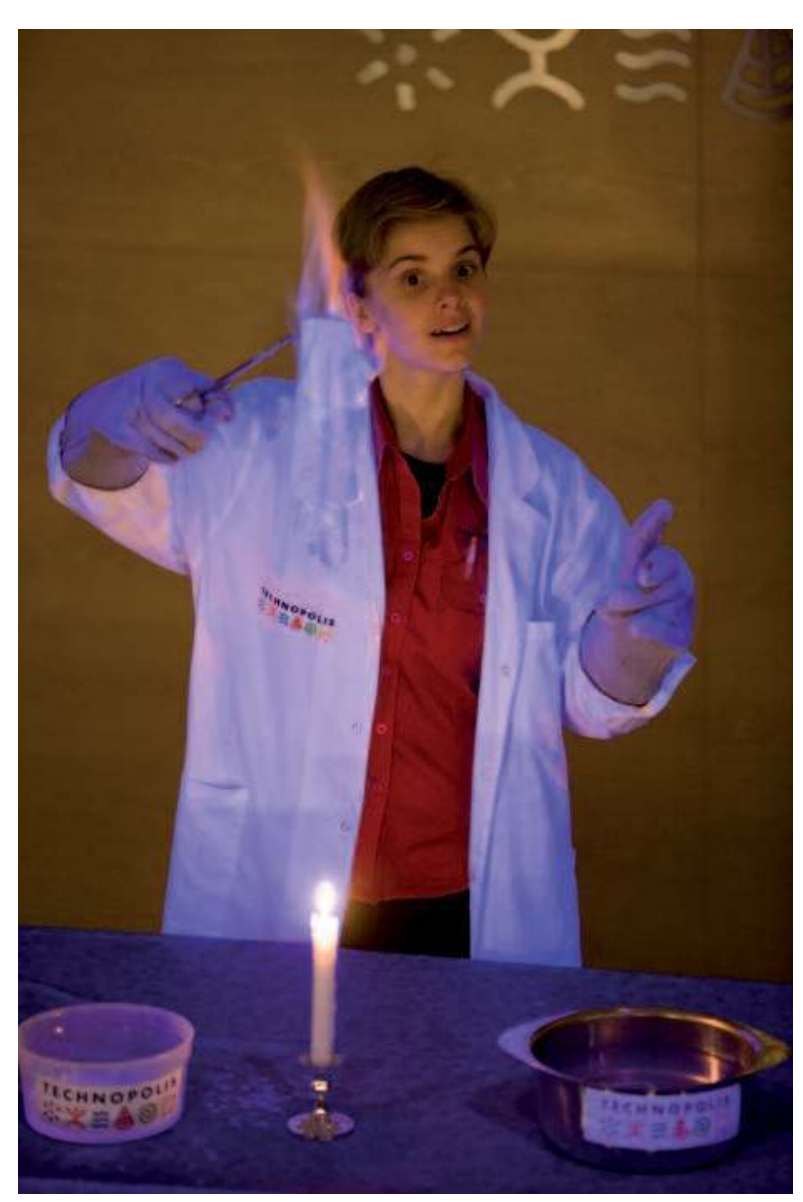

Une médiatrice de Technopolis en cours de démonstration (c) Creative Commons PILOTS

* Olivier Richard est chargé de programmation, département Action Culturelle, Universcience olivier.richard@universcience.fr Sarah Barrett est chargée d'études, Ad Hoc Médiation \& Culture adhoc.culture@laposte.net
Face à la méconnaissance du métier de médiateur scientifique, le projet PILOTS présenté ici - a pour principaux objectifs de dresser un tableau de la diversité des statuts du médiateur scientifique en Europe, de préciser le rôle de cette profession au sein des différentes institutions muséales et d'évaluer ses besoins en formation.

Le concept de médiation a trouvé un terreau fertile dans les musées et particulièrement ceux du champ de la culture scientifique, comme le souligne Paul Rasse : "le succès et l'essor du projet de médiation viendront moins du domaine des arts, que de celui des sciences, dans le sillage du mouvement de mise en culture des sciences et des techniques ». Le médiateur a investi le musée, en lieu et place de l'animateur, dont la dénomination, trop associée au mouvement d'éducation populaire, a été rejetée par les acteurs culturels. Il disparaît ainsi au profit du médiateur scientifique, voire du scientifique médiateur ${ }^{(1)}$. Ce jeu sémantique illustre la reconnaissance d'un métier destiné à réduire la distance (si ce n'est la tension) entre le public et les œuvres, et réaffirme la place du public au coeur des projets culturels. Toutefois, loin des discours qui dressent le portrait du médiateur en «militant du changement social » (J. Hurstel), ou plus simplement en « passeur » (É. Caillet), la réalité ne semble pas toujours attribuer aux médiateurs une place aussi centrale au sein des institutions. Le métier n'incarne pas forcément toutes les intentions du concept, comme le remarque Nicolas Aubouin dans l'étude sur l'évaluation des emplois de la médiation dans le secteur culturel : "les musées ont été les lieux d'une invention locale d'activités et de concepts de médiation. [..] Un tel parcours est aussi rythmé en creux par la 
série des difficultés de traduction de considérations générales en dispositifs opérationnels ». Si la médiation constitue un riche sujet d'études, le médiateur et son activité professionnelle au sein des musées restent un champ encore peu parcouru. Pour envisager cette problématique à l'échelle européenne, six partenaires se sont regroupés au sein du projet PILOTS. Dans ce cadre, une large enquête a cherché à mieux connaître les profils, le rôle et les besoins des médiateurs des musées et centres de sciences européens. Elle s'est organisée en deux phases, la première constituée d'une série de focus-groups organisés dans les différents musées partenaires, la seconde à travers une enquête quantitative en ligne.

Des groupes de discussions ont été organisés dans chacun des musées participants au projet, en France, Italie, Belgique, Slovénie et Portugal. Les groupes, composés de médiateurs de la même institution, devaient aborder une série de thèmes liés à leurs pratiques professionnelles. Une grille commune à tous, élaborée par les évaluateurs, organisait les échanges et permettait une lecture comparée des résultats. Les échanges se faisaient naturellement dans la langue de chaque pays, permettant ainsi des discussions approfondies. L'usage de l'anglais aurait simplifié l'analyse, mais s'accompagnait de trop d'effets négatifs, comme l'impossibilité de participer pour des médiateurs non anglophones ou encore le risque de débats sans profondeur. Lanalyse des focusgroups a permis de dresser un premier tableau des enjeux liés à la diversité des médiateurs scientifiques dans les différentes institutions européennes, mettant en évidence ce qui distingue mais aussi ce qui rassemble les représentants de cette profession aux contours mal connus. La seconde phase de l'étude visait à compléter les informations recueillies lors des focus-groups grâce une enquête quantitative auprès d'un échantillon élargi de médiateurs européens. Elle portait plus particulièrement

\section{PILOTS (Professionalisation in Learning Technology and Science)}

Projet européen, soutenu par le programme pour la Formation Continue (DG Culture \& Éducation) et placé sous la coordination générale d'Ecsite, PILOTS a regroupé pendant 2 ans (novembre 2008 à octobre 2010) six partenaires européens :

- quatre centres de sciences : Technopolis (Malines, Belgique), Universcience (Paris, France), Ciência Viva (Lisbonne, Portugal) et Ustanova Hisa eksperimentov (Lubjana, Slovénie)

- un musée scientifique et technique : Fundazione Museo Nazionale della Scienza et della Technologica «Leonardo da Vinci » (Milan, Italie)

- un laboratoire de recherche en communication scientifique : SISSA Medialab (Trieste, Italie)

Le projet visait à contribuer à l'évolution des modes d'interactions des médiateurs scientifiques avec le public adulte, en impliquant les institutions et la communauté des médiateurs scientifiques à l'échelle européenne. Il poursuivait deux objectifs :

- la collecte d'informations sur les profils et rôles des médiateurs scientifiques au niveau européen. Les rapports complets des études menées sont disponibles en français auprès des auteurs et en anglais sur le site du projet;

- la conception et le test de modules de formation mettant en exergue le rôle des médiateurs scientifiques dans l'apprentissage informel des adultes. Ces modules se sont plus particulièrement focalisés sur les nouveaux besoins des médiateurs, pour faciliter le dialogue et les échanges avec les visiteurs, aborder des sujets sensibles et prêtant à controverses, prendre en compte les différences de genres ou encore présenter la recherche actuelle. Quatre sessions de tests organisées à Malines, Milan, Paris et Dortmund ont permis d'évaluer et d'améliorer les formations au cours du projet.

L'ensemble des modules de formation conçus sont disponibles sur le site du projet : www.thepilots.eu. Une plateforme Internet (le « Hub Pilots », un réseau social professionnel) a également été développée et mise à disposition de la communauté des médiateurs européens. (http://pilots-hub.ning.com).

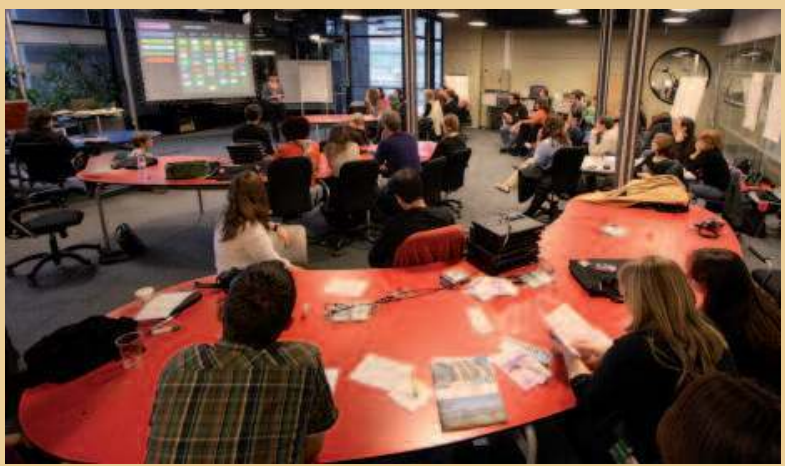

Formation des médiateurs lors de la session PILOTS accueillie à Universcience à Paris en janvier 2010. (c) Creative Commons PILOTS 
sur les profils des médiateurs scientifiques qui travaillent au sein des institutions européennes, ainsi que sur leurs rôles et leurs besoins en formation. Pour cela, un questionnaire était accessible sur Internet de juillet 2009 à février 2010. Les médiateurs ont été contactés via le récent réseau social européen créé à l'initiative de PILOTS et hébergé sur le site Internet du projet, ainsi que grâce à une invitation envoyée aux institutions membres des réseaux professionnels européens (Ecsite) et nationaux impliqués. 236 questionnaires ont ainsi été recueillis et analysés.

\section{Des médiateurs diplômés majoritairement féminins}

Les résultats montrent que, si pour les plus jeunes la fonction de médiateur scientifique reste un emploi occupé le temps des études, il s'agit pour d'autres plus âgés d'une véritable profession dans laquelle ils s'installent. L'âge moyen des médiateurs européens dépasse 30 ans, et la majorité sont titulaires d'emplois permanents (et près des trois-quarts pour ceux âgés de plus de trente ans).

Phénomène remarquable : le haut niveau de diplôme des médiateurs interrogés. Plus de la moitié d'entre eux détiennent un master (38\%) ou un doctorat (14\%). Au total en comptant également les managers, $57 \%$ des professionnels de la médiation scientifique sont titulaires d'un diplôme de niveau master ou doctorat. Par comparaison ce taux au sein de l'Union Européenne est de $28 \%$ chez les 25-34 ans. L'écart apparaît encore plus caractéristique pour les seuls détenteurs de doctorats : moins de $1 \%$ au sein de l'Union Européenne, ils sont $17 \%$ chez les professionnels de la médiation des musées de sciences. La carrière de médiateur s’inscrit donc dans la continuité des études supérieures.

Parmi ces médiateurs très diplômés, il s'avère également que plus des deux tiers sont des femmes, alors qu'une parité est observée chez les détenteurs de diplômes moins élevés.

Au sein de l'Union Européenne, 44 \% des diplômés de l'enseignement supérieur sont des femmes. Pour les pays plus particulièrement représentés dans l'étude PILOTS, ce taux varie de $37 \%$ en Belgique à $51 \%$ en Italie, mais il n'atteint jamais le déséquilibre observé chez les médiateurs des musées européens. Cette situation semble être une conséquence directe de la seconde grande préoccupation européenne concernant l'égalité des genres dans l'enseignement supérieur : la ségrégation verticale. "Ce problème est lié au plafond de verre qui existe actuellement dans l'enseignement supérieur: alors que les

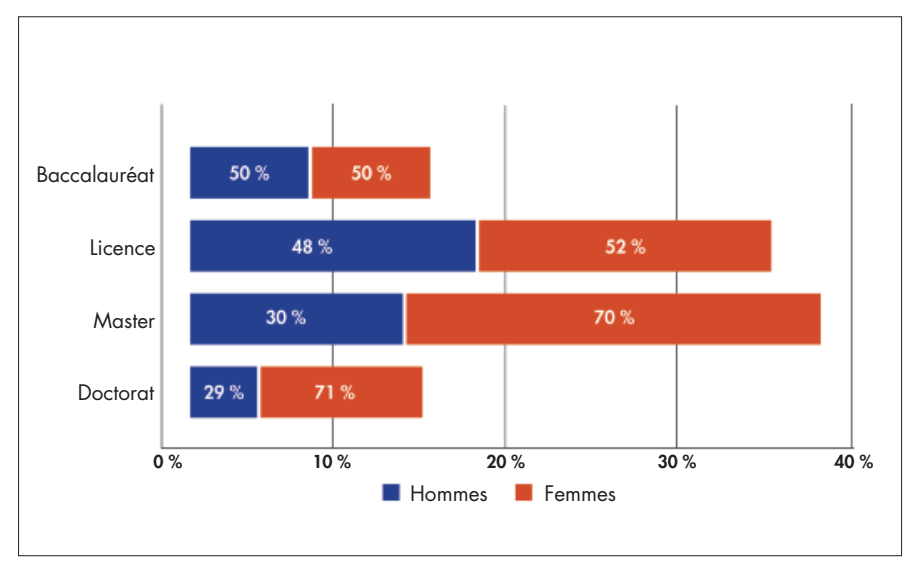

Niveau de diplôme des médiateurs selon le genre

femmes sont plus nombreuses que les hommes parmi les diplômés de l'enseignement supérieur, elles sont légèrement sous représentées dans les études de doctorat, et parmi le personnel académique des universités », précise l'EACEA (agence exécutive pour l'éducation, l'audiovisuel et la culture en Europe). Les femmes sont moins nombreuses que les hommes à pouvoir accéder aux postes de chercheurs, et la situation en France lillustre bien : en 2005 les femmes représentaient $55 \%$ des étudiants à l'entrée de l'université, mais seulement $40 \%$ des titulaires d'un doctorat et $30 \%$ des recrutements comme chercheur ou enseignant à l'université. Le corps des professeurs et directeurs de recherche comptait $15 \%$ de femmes (Source : ministère de l'Enseignement supérieur et de la Recherche).

\section{Le credo du médiateur : polyvalence et adaptation}

L'absence de formation spécifique à l'échelle européenne à cette profession entraîne des profils de médiateurs très divers dans les différents centres étudiés. Chaque structure définit ses propres critères de sélection. Le profil des médiateurs va ainsi de l'étudiant au chercheur scientifique. De plus, les formations internes des musées étudiés présentent la même hétérogénéité.

Les médiateurs doivent également se montrer d'une grande adaptabilité dans leurs tâches quotidiennes. En une journée, ils jonglent avec des activités différentes et ils sautent sans transition de la conception d'activités à l'interaction avec le public. Par conséquent, il est évident que les médiateurs doivent être plein de ressources et mobiliser pour cela une diversité de compétences scientifiques, relationnelles, artistiques et organisationnelles. La polyvalence semble donc être le credo de cette profession. Nathalie Montoya relève que «cette question se traduit concrètement chez les médiateurs dans la formulation du dilemme qui 


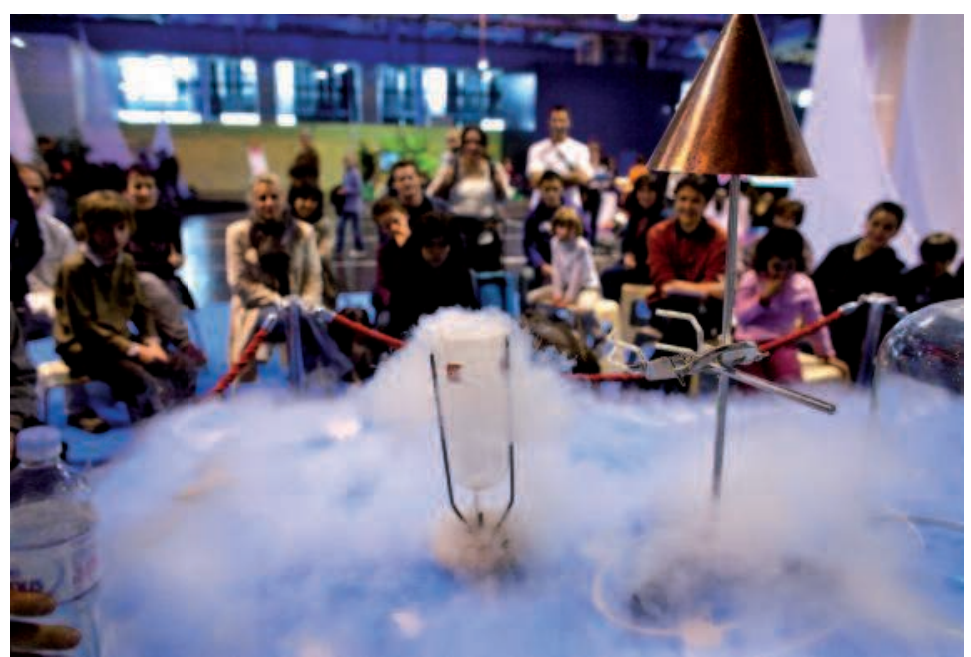

Les médiateurs de la Cité des Sciences et de l'Industrie en action dans Explora ๑ CSI/A. Robin

oppose activités de terrain (supposées proches du public) et activités de bureau (supposées proches du coeur créatif du métier parce qu'elles contiennent une part de conception importante) ». Dilemme qui apparaît clairement dans l'étude menée au niveau européen, dans laquelle la conception et la gestion de projet constituent les dimensions du métier considérées par les médiateurs comme ce qu'ils voudraient plus souvent faire ou pour lesquelles ils souhaiteraient plus de reconnaissance.

Si les médiateurs décrivent le «sens » de leur travail, sa «portée » de façon assez similaire dans les différents centres étudiés, leurs actions sur le terrain, leurs domaines de compétences et leurs interventions auprès du public (mais aussi hors public) diffèrent en revanche significativement. L'absence de consensus dans les activités pratiquées s'illustre dans la très grande diversité de dénominations du métier à travers l'Europe, obligeant même l'usage de néologismes pour les traduire fidèlement : moniteur, animateur, guide, opérateur, « explicateur », présentateur, interprète, démonstrateur, « interacteur », facilitateur, présentateur, hôtesse, médiateur, animateur, modérateur, informateur, « médiacteur », sans oublier les très expressifs « edutainer » ou «phenoman ». En cela, la notion de médiateur scientifique semble se référer à un ensemble d'activités plutôt qu'à une profession précise. Si la médiation est une fonction, il n'est pas évident qu'elle s'incarne dans un métier unique.

À travers leurs témoignages, les médiateurs se définissent eux-mêmes comme un «complément humain » de l'exposition, voire parfois comme un «média humain». Ils apportent des précisions et des explications qui complétent l'exposition avec une plus-value fondamentale : ils interagissent avec le public. En bref, leur principale fonction consiste à aider le public dans sa démarche de découverte et de questionnement. Ainsi, s'il n'y a pas de consensus sur le nom de la profession, il est important de souligner que tous sont unanimes sur la définition de leur rôle. En interface entre l'exposition et les visiteurs, leur mission telle quilils la définissent est de rendre accessibles et compréhensibles des faits scientifiques de façon ludique à une diversité de gens. Il s'agit de «démocratiser » la science et de la transmettre aux publics, en la traduisant afin que ces derniers comprennent au mieux les informations. Un médiateur résume ainsi ce rôle : "Pour moi, le rôle du médiateur ça doit être de faire comprendre au plus grand nombre un truc scientifique. C'est un peu comme un diplomate. Les sciences ça fait peur aux gens, et grâce à nous, j'espère, on arrive à montrer la science sous un autre jour. Et à rendre ça intelligent, intelligible».

Une transmission ludique du savoir, mais surtout du désir de comprendre en stimulant la curiosité du public. Les médiateurs décrivent leur rôle en insistant sur le fait qu'ils souhaitent "éveiller la curiosité ", non seulement pendant la visite, mais aussi à long terme. Beaucoup déclarent que leur plus grande satisfaction serait de savoir que les visiteurs continuent de penser au sujet quand ils retournent chez eux et qu'ils recherchent des informations complémentaires sur les questions abordées lors de leur visite.

Le cœur du métier de médiateur reste principalement l'accompagnement du public, dans les expositions ou au cours d'ateliers, comme le souligne un médiateur interrogé : "Le temps en face public est quand même important et c'est à mon avis ce qui est un peu le centre de ce qu'on fait. C'est-à-dire que tout ce qu'on fait c'est pour ce moment-là normalement ». Les médiateurs scientifiques se représentent eux-mêmes comme des «bateleurs scientifiques ", image partagée par leurs managers. Les activités de conception et de coordination de projets, également très présentes, sont toutefois assurées par une part moins importante des médiateurs. Mais la répartition des types de tâches semble se faire sans distinction d'âge, de diplôme, de catégorie d'emploi ou d'ancienneté dans le poste : les activités de conception ne sont pas réservées aux plus anciens ou aux plus diplômés. Il s'agit plutôt d'une logique cumulative, où certaines tâches s'ajoutent au cœur du métier qui reste principalement l'accompagnement du public, dans les expositions ou au cours d'ateliers. Ce public accueilli par les médiateurs scientifiques est avant tout familial. Mais au-delà du public le plus jeune, les médiateurs comme leurs managers reconnaissent unanimement 
que s'adresser au public adulte requiert des compétences spécifiques, sans que cela ne se traduise par une forme de médiation qui serait plus particulièrement développée pour les adultes.

\section{Des besoins en formations peu entendus}

À cette unanimité des représentations du métier de médiateur s'opposent les visions divergentes en matière de formations. Ainsi, $60 \%$ des managers indiquent proposer des formations plus ou moins longues lorsque les médiateurs débutent leur emploi, mais seulement $25 \%$ de ceux-ci déclarent en avoir bénéficié.

Une liste identique de 22 compétences caractéristiques du métier de médiateur a été proposée à la fois aux médiateurs et à leurs managers. Cette liste repose notamment sur les travaux très complets menés par Cortex Culturemploi sur les emplois culturels (www.cortex-culturemploi.com) et Isabelle Mathieu (Mode d'emploi culturel : les métiers de la culture, Paris : Éditions Weka, 2008).

Interagir avec les visiteurs / Parler en public / Travailler en groupe / Avoir un intérêt pour la science / Transmettre des connaissances / Adapter son discours selon les visiteurs / Faire participer le public / Être créatif / Concevoir une animation / Travailler avec des partenaires éducatifs / Travailler avec des bénévoles ou des sous-traitants / Diriger un groupe de travail / Connaître la sociologie des publics / Coordonner un projet / Avoir une maîtrise dans une ou plusieurs disciplines scientifiques / Suivre la maintenance du matériel / Connaître les acteurs de la culture scientifique / Écrire un texte de vulgarisation / Organiser une formation / Établir des partenariats / Conduire une évaluation / Concevoir des expositions /

Les managers devaient choisir dans cette liste les dix propositions qu'ils estimaient être les plus importantes pour être un bon médiateur. De leur côté, les médiateurs devaient préciser pour chacune des 22 mêmes compétences, si ils les considéraient comme déjà acquises, si elles étaient nécessaires mais demandaient une formation complémentaire ou si elles s'avéraient simplement inutiles dans le cadre de leurs fonctions. Les points de vue des médiateurs et de leurs managers ont pu ainsi être comparés pour chacune des compétences proposées, selon l'importance relative donnée par les managers à chacune d'entre-elles et le degré d'utilité ou le besoin en formation déclaré par les médiateurs.
La comparaison des deux classements obtenus selon l'importance relative donnée par les managers d'une part et le besoin de formation déclaré par les médiateurs d'autre part fait apparaître quatre grandes catégories de compétences.

\section{Les compétences évidentes}

Il s'agit ici des compétences fondamentales de la profession, principalement axées sur les modes de communication avec le public : interagir avec les visiteurs, adapter son discours selon les visiteurs, être créatif, transmettre des connaissances, parler en public, avoir un intérêt pour la science, travailler en groupe, faire participer le public. Elles sont jugées à la fois importantes par une majorité des managers (plus de $65 \%$ ), et demandant une formation complémentaire par une minorité de médiateurs (15 à $30 \%$ d'entre eux). Plus de $60 \%$ des médiateurs les considèrent par ailleurs comme déjà acquises. Si une offre en formation reste nécessaire, notamment pour les médiateurs " juniors ", les demandes majeures en formations portent sur les autres aspects du métier.

\section{Les compétences nécessaires}

Ces compétences sont moins que les premières axées sur la prestation en public et abordent la face cachée du travail des médiateurs, comme connaître la sociologie des publics ou travailler avec des partenaires éducatifs. Ces aspects pour lesquels une majorité de médiateurs demande des formations complémentaires, sont jugés comme importants par la moitié des managers. Une part importante des médiateurs considère détenir déjà ces compétences, et $90 \%$ d'entre eux les jugent nécessaires.

\section{Les compétences non reconnues}

Cette catégorie regroupe les compétences pour lesquelles la majorité des médiateurs demande des formations complémentaires (entre 50 et $60 \%$ d'entre eux). Les besoins affichés se rapportent plus globalement aux champs de la conception et de la gestion de projets : coordonner un projet, diriger un groupe de travail, écrire un texte de vulgarisation, organiser une formation ou conduire une évaluation. Elles concernent également les relations des institutions avec les autres acteurs du champ de la culture scientifique et du secteur muséal : « connaître les acteurs de la culture scientifique » ou «établir des partenariats ». Ces fonctions sont toutefois considérées comme importantes par une minorité de managers (moins de $30 \%$, et le plus souvent moins de $20 \%$ ), peut-être parce que relevant plus directement de leur propre cœur de métier. 


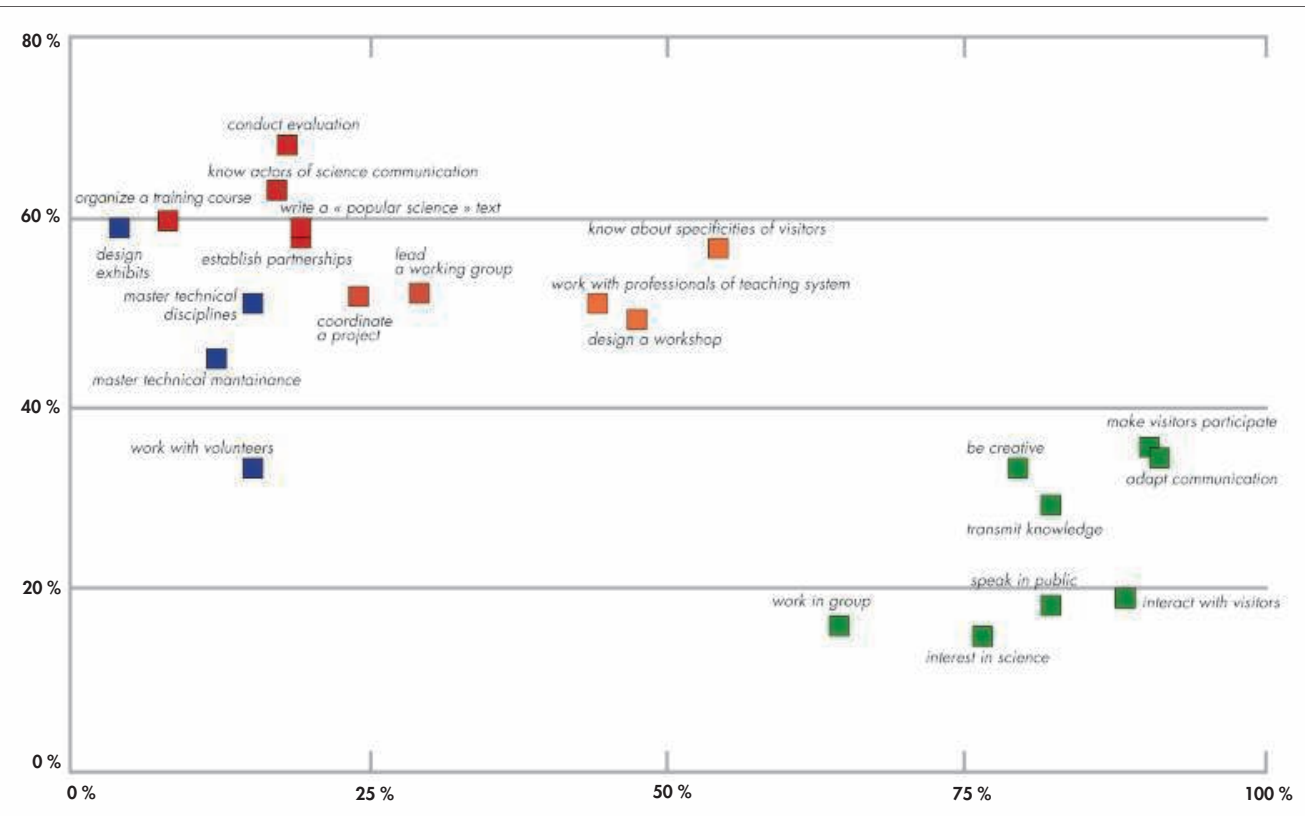

Compétences classées selon leur besoin de formation déclaré par les médiateurs (vertical) et leur degré d'importance pour leurs managers (horizontal). Exemple de lecture : «Savoir parler en public » est une compétence que $82 \%$ des managers classent parmi les 10 plus importantes et pour laquelle $18 \%$ des médiateurs demandent une formation complémentaire.

Les compétences marginales

Cette dernière catégorie se compose de compétences jugées comme étant les moins importantes par les managers (moins de $15 \%$ d'entre eux les citent parmi les 10 plus importantes) et peu nécessaires par les médiateurs (plus de $25 \%$ d'entre eux les jugent inutiles). Il s'agit de fonctions qui paraissent marginales dans l'exercice quotidien du métier de médiateur : travailler avec des bénévoles ou des sous-traitants, suivre la maintenance du matériel. La question de la conception des expositions l'illustre parfaitement : fonction quasi-unanimement considérée par les managers comme extérieure au métier de médiateur $(4 \%$ de citations seulement parmi les 10 fonctions importantes), un quart des médiateurs la juge également inutile, soit un des taux les plus forts observés sur ce critère de la nécessité.

Si les compétences "évidentes », essentiellement liées aux activités avec le public et au cœur de métier, (mais pour lesquelles les médiateurs se considèrent majoritairement formés) doivent naturellement continuer à faire l'objet de formations, les enjeux essentiels portent in fine sur les compétences qui recueillent en effet la plus forte demande en formation de la part des médiateurs. Ainsi, l'ensemble des compétences liées au développement d'activités et plus globalement à la gestion de projet sont essentielles à la structuration et la professionnalisation du médiateur, auxquelles s’adjoignent les connaissances plus générales sur le contexte professionnel, la connaissance des acteurs de la communication scientifique comme celle des caractéristiques du public.

Ces activités « de bureau » semblent toutefois rester invisibles pour les managers, qui conçoivent d'abord et avant tout le médiateur face à son public. Il convient d'identifier parmi les demandes des médiateurs celles qui correspondent effectivement aux fonctions attendues et aux besoins réels des institutions. La question de la conception des expositions en est une bonne illustration : si près de 3 médiateurs sur 5

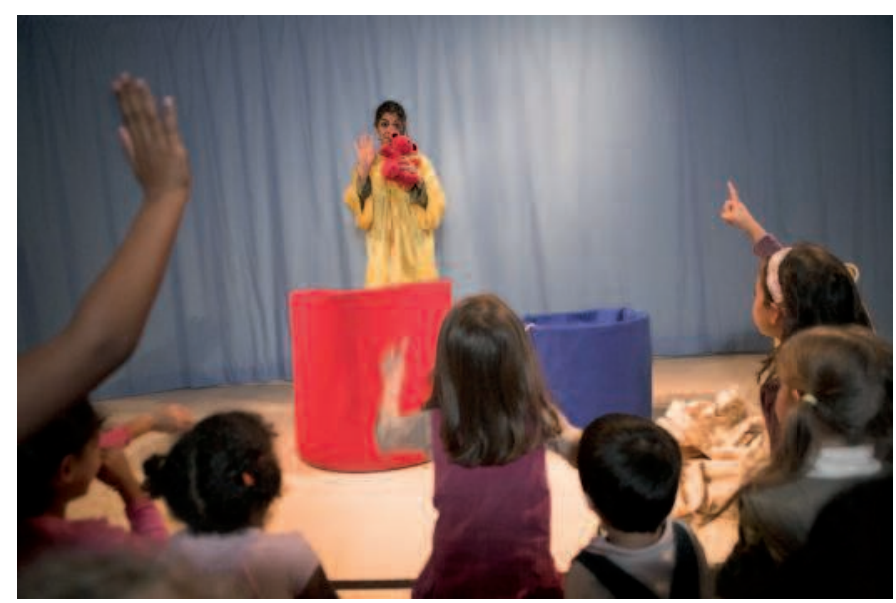

Un atelier/spectacle à la Cité des Enfants () CSI/E. Bartolucci 
désirent y être formés, les managers ne considèrent pas que cette compétence soit associée à la fonction de médiateur, mais qu'elle constitue en fait un métier à part au sein du musée. Toutefois, la conception d'expositions semble représenter pour les médiateurs un espoir, si ce n'est le seul, d'ascension professionnelle au sein de l'institution. Cette perception, in fine dommageable aux activités de médiation, révèle en creux le besoin de reconnaissance dont souffre l'ensemble de la communauté des médiateurs au sein de leurs structures.

\section{Un manque de reconnaissance partagé à l'échelle européenne}

La grande diversité observée tant du point de vue des profils des personnes que des tâches accomplies engendre une relative dévaluation de la profession, allant parfois jusqu'à une impression de stagnation professionnelle, une impossibilité d'évoluer dans son métier, voire une absence totale de perspectives. Cela rejoint le constat fait par Nathalie Montoya sur le manque de visibilité de la médiation, qu'elle attribue pour sa part à la nature même de l'action : "La signification du terme «médiation» engage une relation ambivalente au visible. Si le médiateur vise à relier deux termes entre eux, il tend également à s'effacer devant l'établissement de ce lien. La tendance de la médiation, quel que soit son champ d'application, est d'abolir l'écart ou de résoudre le conflit qui fondait la nécessité de son intervention, et de ce fait, de disparaitre dans sa propre réalisation ». Nathalie Montoya en pointe justement les conséquences : "Cette faible visibilité de la médiation a des répercussions sur la reconnaissance au sein même des institutions de la nécessité de cette activité. Que le besoin de médiation ne se fasse sentir que lorsquielle manque conduit parfois les médiateurs à vivre leur métier comme une tâche fatalement ingrate, qui n'est reconnue que lorsqu'elle faillit à sa mission ». Tout cela participe in fine à l'absence d'un véritable « esprit de corps » lié au métier et d'une véritable reconnaissance. Ceci pose la question de savoir si être médiateur est moins une profession dans laquelle il est possible de faire carrière qu'une activité transitoire, la porte d'entrée du musée si ce n'est le sas pour espérer gagner les étages de la reconnaissance professionnelle, souvent espérée dans la sphère de la conception des expositions.

Malgré leur niveau de diplôme élevé, les médiateurs restent ainsi perçus au sein des musées principalement à travers leurs tâches d'accompagnement du public. Il convient néanmoins de poursuivre la construction d'une image du métier de médiateur plus complexe en valorisant et développant les compétences liées à la conception de projet, mais aussi plus dynamique en interrogeant plus profondément les logiques de carrière actuellement à l'œuvre, tant dans le choix du métier que dans les perspectives d'évolution professionnelle offertes au sein (ou en dehors) des institutions.

Cette image des médiateurs est avant tout celle que l'institution a conçue et renvoie. Le musée ne laisse que peu de place aux activités de conception et de gestion dans l'idée qu'il se fait du métier de médiateur. Alors même que l'activité «face public » ne constitue que la partie émergée de la réalité du métier, sans doute minoritaire en termes de temps de travail cumulé, le musée continue de présenter l'activité de médiateur comme étant exclusivement en action avec le public. Les autres dimensions sont dans le meilleur des cas minimisées, le plus souvent tues. C'est donc tout d'abord à l'intérieur même du musée que l'image réductrice de la fonction du médiateur semble se forger. La question des publics, du rapport au public et de sa connaissance, a priori déterminante dans l'activité de médiation, illustre ce paradoxe. Comme le rapporte Nathalie Montoya, «les médiateurs se confrontent encore rarement à l'enchevêtrement des pratiques et des expériences de leurs publics. Les médiateurs culturels ont tendance à construire des représentations monolithiques de l'expérience esthétique et du rapport à la culture ». Ce constat lapidaire est partagé par les médiateurs, qui soulignent unanimement la nécessité de cette compétence : seuls $4 \%$ d'entre eux la jugent inutile et une majorité demande une formation complémentaire sur ce sujet. Les managers sont nettement moins unanimes et une partie seulement d'entre eux classe cette compétence parmi les dix principales.

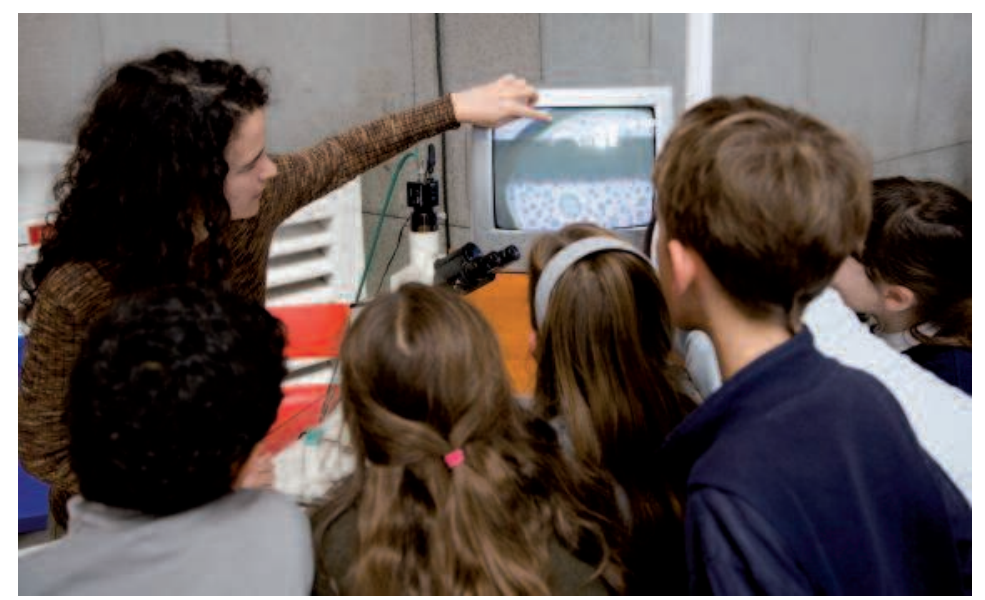

Un atelier réalisé à la Cité des Sciences et de l'Industrie lors de la Fête de la Science. () CSI/S. Chivet 


\section{Conclusion}

L'étude européenne PILOTS constitue une première observation à cette échelle et offre une série de résultats tant quantitatifs que qualitatifs sur la réalité du métier au sein des musées et centres de sciences européens. Elle dresse un panorama élargi de l'action quotidienne des médiateurs, tant en présence du public que dans les moments moins visibles mais tout aussi nécessaires de conception et de gestion de projets. Si la polyvalence reste le maitre mot du métier, il conviendrait d'approfondir cette question en travaillant sur un répertoire de compétences partagé au niveau européen, à l'image de certaines initiatives à l'échelle des métiers des musées, afin de mieux connaître, unifier et donner un cadre précis à la profession de médiateur pour favoriser une plus grande reconnaissance au sein des institutions culturelles.

Une différence de représentation du métier apparaît en effet clairement entre les médiateurs et leurs managers. Ces derniers envisagent la figure du médiateur essentiellement en présence du public, ignorant l'ensemble des tâches de conception et de coordination de projets. S'il convient de poursuivre les efforts de formation autour des compétences évidentes que tout médiateur doit maîtriser pour s'adresser au public, un accent particulier devra être porté sur celles tout aussi indispensables à l'exercice quotidien du métier, comme la gestion de projet ou la connaissance des publics.

Certaines dimensions comme les dynamiques de carrière restent encore à explorer. Il conviendrait pour cela de compléter cet état des lieux en intégrant une vision dynamique des parcours professionnels des médiateurs. En premier lieu en étudiant plus finement les itinéraires initiaux, à savoir identifier les motivations des médiateurs choisissant d'exercer ce métier, notamment en fonction des parcours universitaires préalables et des choix effectués, par défaut ou non. En second lieu, en déterminant les évolutions professionnelles potentielles au sein des institutions ou en dehors à partir des parcours et carrières d'anciens médiateurs.
C'est essentiellement à l'intérieur même du champ des musées qu'il convient de travailler pour combattre les préjugés et les idées reçues véhiculées par et dans les institutions elles-mêmes. Sensibiliser les cadres d'une part, mais aussi encourager les échanges de médiateurs entre les structures, nationales comme européennes, conditions indispensables à l'émergence d'un véritable esprit de corps professionnel.

\section{Note}

(1) La référence à la médiation semble à son tour être de moins en moins utilisée, comme le note Nicolas Aubouin : «le terme « médiateur » est de moins en moins utilisé par les acteurs pour qualifier leur activité. [...] Bien que le qualificatif " médiateur "soit aujourd'hui récusé par nombre des acteurs, notamment en raison de son héritage "emploi-jeune ", il reste parfaitement intelligible pour eux ». Arbouin, N., Kletz, F. et Lenay, O. Entre continent et archipel, les configurations professionnelles de la médiation culturelle, document de travail du DEPS, Paris, octobre 2009. Voir également

EACEA and Eurydice, Différences entre les genres en matière de réussite scolaire: étude sur les mesures prises et la situation actuelle en Europe, 2010. Mathieu, I. L'introuvable médiateur culturel, in Les processus de construction identitaire en Sciences de l'Information-Communication, 2009.

Montoya, N. Médiation et médiateurs culturels : quelques problèmes de définition dans la construction d'une activité professionnelle, Lien social et Politiques, n60, 2008, pp. 25-35.

Rasse, P. La médiation, entre idéal théorique et application pratique, Recherche en communication, $\mathrm{n}^{\circ} 13,2000$. 